\title{
Diagnostic value of various biochemical parameters for the diagnosis of pheochromocytoma in patients with adrenal mass
}

\author{
Nicole Unger ${ }^{1}$, Christian Pitt ${ }^{4}$, Ingo Lopez Schmidt ${ }^{1}$, Martin K Walz ${ }^{4}$, Kurt W Schmid ${ }^{3}$, Thomas Philipp ${ }^{2}$, \\ Klaus Mann ${ }^{1}$ and Stephan Petersenn ${ }^{1}$ \\ Divisions of ${ }^{1}$ Endocrinology, and ${ }^{2}$ Nephrology and Hypertension, Medical Centre, University of Essen, hufelandstr. 55 , 45122 Essen, Germany, \\ ${ }^{3}$ Institute of Pathology, University of Essen, Essen, Germany and ${ }^{4}$ Department of Surgery and Center of Minimally Invasive Surgery, Kliniken Essen- \\ Mitte, University of Essen, Essen, Germany \\ (Correspondence should be addressed to S Petersenn; Email: stephan.petersenn@uni-essen.de)
}

\begin{abstract}
Objective: Pheochromocytomas are neoplasms generally characterized by the autonomous production of catecholamines. This study compared various biochemical parameters for the diagnosis of adrenal pheochromocytoma in patients with adrenal mass.

Design: One hundred and fifty subjects were studied, including 24 histologically proven pheochromocytomas, 17 aldosterone-secreting and 21 cortisol-secreting adrenal adenomas and 30 nonfunctioning adrenal masses, 16 patients with essential hypertension and 42 healthy normotensive volunteers. Spontaneous blood samples and 24-h urine samples were collected prospectively.

Methods: Plasma and urinary epinephrine and norepinephrine levels were measured by high performance liquid chromatography, whereas plasma and urinary metanephrine and normetanephrine levels were determined by radioimmunoassay (RIA). Putative ratio thresholds were calculated by receiver operating characteristic (ROC) analysis to balance between sensitivity and specificity.

Results: Plasma normetanephrine was found to be the best single parameter with the highest sensitivity $(91.7 \%)$ and specificity $(95.6 \%)$ using a threshold of $126 \mathrm{pg} / \mathrm{ml}$. In combination, plasma normetanephrine and metanephrine had a higher sensitivity of $95.8 \%$ with lower specificity $(79.4 \%)$. All other combinations of plasma and/or urinary parameters demonstrated a lower accuracy. Conclusion: Plasma metanephrines measured by RIA are reliable screening parameters for the diagnosis of pheochromocytoma.
\end{abstract}

European Journal of Endocrinology $15 \mathbf{5 4} 409-417$

\section{Introduction}

Pheochromocytomas are catecholamine-producing tumors arising from chromaffin cells of the adrenal medulla or extra-adrenal paraganglionic tissue (1). The inappropriate concentration of catecholamines may result in sustained or intermittent severe hypertension. Typical clinical symptoms are headache, sweating and tachycardia. Due to improved magnetic resonance imaging (MRI) or computed tomography (CT) techniques and a frequent use of these tools, adrenal tumors are increasingly recognized. Accurate biochemical parameters are required to discriminate pheochromocytomas from other hormone secreting or inactive adrenal adenomas. An undiagnosed pheochromocytoma might result in life-threatening consequences. Therefore, highly sensitive biochemical assays are essential to avoid false-negative results. The diagnosis of pheochromocytoma is based on the overproduction of catecholamines. Determinations of plasma and in particular 24-h urinary epinephrine and norepinephrine levels are established diagnostic tools. A handicap is that they may be falsely negative in patients with a biochemically silent or periodically secreting pheochromocytoma (2). On the other hand, they may be falsely elevated in patients with panic disorder or congestive heart failure (2). Metanephrines, which are metabolites of catecholamines, have been suggested as an alternative diagnostic tool (3-5). The aim of this study was to establish thresholds for plasma and urinary catecholamines and metanephrines with optimal sensitivity and specificity for the diagnosis of pheochromocytoma in patients with an adrenal mass. Patients with hereditary syndrome were not considered. Recently, a radioimmunoassay has been developed, which was employed in our study to determine plasma and urinary metanephrine and normetanephrine. 


\section{Methods}

\section{Subjects}

Ninety-two subjects were prospectively included in this study. Inclusion criterion was the presence of an adrenal mass requiring an operation. The patients were grouped into four categories: 24 adrenal pheochromocytomas (PHEO), 17 aldosterone-secreting adenomas (APA), 21 cortisol-secreting adenomas (CPA) and 30 nonfunctioning adrenal masses (NFM; twenty nonfunctioning adenomas (NFA), two cysts, three myelolipoma, one adrenal carcinoma, one ganglioneurinoma, one T-cell-lymphoma, one metastasis of a bronchial carcinoma and one of a hypernephroma). Controls included 16 patients with essential hypertension and 42 healthy normotensive volunteers (pertinent data are given in Table 1). In patients with essential hypertension, a pheochromocytoma was considered unlikely due to the absence of specific clinical symptoms and adrenal tumors in MRI scans. Healthy volunteers presented a normal blood pressure on three different occasions. Therefore, no further procedures were carried out to exclude a pheochromocytoma. Pheochromocytomas were diagnosed preoperatively by endocrine testing, MRI or CT and ${ }^{123}$ I-meta-iodobenzylguanidine scintigraphy. The diagnoses were postoperatively confirmed by histological and immunohistochemical examinations. All pheochromocytomas were considered benign at the time of diagnosis. Proof of malignancy was the appearance of tumor cells in non-chromaffin tissue or local tumor invasion (6). All pheochromocytomas were considered as sporadic. The family history of the patients investigated did not point to a familial pheochromocytoma. Genetic testing was carried out for MEN2, VHL, and mutations of the succinate dehydrogenase complex, subunit B (SDHB) gene and succinate dehydrogenase complex, subunit D (SDHD) gene. However, we cannot completely exclude a genetic syndrome without family history.

The etiology of the remaining adrenal masses was preoperatively established by respective biochemical parameters and MRI or CT. All adrenal masses were subsequently removed and the diagnosis was postoperatively confirmed by histological examinations as well as by clinical follow-up. At the time of investigation, patients were on various antihypertensive medications including beta-blockers as well as central and peripheral alpha-blockers. Blood samples were drawn in a seated body position with standard venipuncture from a forearm vein. Urine samples were collected in vessels containing $\mathrm{HCl}$. Blood and urine samples were obtained within the same day. All samples were collected prospectively from June 2002 to June 2004 and were analyzed by the same investigator blinded to the clinical condition of the patients. To test the reproducibility of the plasma metanephrine and normetanephrine measurements within individuals, blood samples from nine healthy volunteers (3 male, 6 female) were collected on three consecutive days. Mean age ( \pm S.E.M.) was $34.6 \pm 3.4$ years. The local ethical committee approved the study protocol and all patients gave informed consent.

\section{Assays}

Plasma and urinary catecholamines were measured by high-performance liquid chromatography (HPLC; Chromsystems, Munich, Germany). Assay sensitivity for plasma catecholamines was $10 \mathrm{pg} / \mathrm{ml}$ each. Assay variability was determined by within-assay coefficients of variation of $5.8 \%$ for $218 \mathrm{pg} / \mathrm{ml}, 5.4 \%$ for $54 \mathrm{pg} / \mathrm{ml}$ and $6.8 \%$ for $47 \mathrm{pg} / \mathrm{ml}$. Assay sensitivity for urinary catecholamines was $1 \mu \mathrm{g} / 24 \mathrm{~h}$ each. Assay variability was determined by within-assay coefficients of variation of $3.7 \%$ for $48 \mu \mathrm{g} / 24 \mathrm{~h}, 4.2 \%$ for $17 \mu \mathrm{g} / 24 \mathrm{~h}$ and $4.4 \%$ for $167 \mu \mathrm{g} / 24 \mathrm{~h}$. Plasma free and urinary metanephrine and normetanephrine were analyzed by RIA (DLD Diagnostika GmbH, Hamburg, Germany). Assay sensitivity was $10 \mathrm{pg} / \mathrm{ml}$ for plasma metanephrine and normetanephrine. Assay variability was determined by coefficients of variation of $6.5 \%$ for $62 \mathrm{pg} / \mathrm{ml}$ and $7.3 \%$ for $713 \mathrm{pg} / \mathrm{ml}$ for plasma metanephrine and $6.9 \%$ for $170 \mathrm{pg} / \mathrm{ml}$ and $7.8 \%$ for $161 \mathrm{pg} / \mathrm{ml}$ for plasma normetanephrine. Between-assay coefficients of variation were $7.8 \%$ for $69 \mathrm{pg} / \mathrm{ml}$ and $8.3 \%$ for $804 \mathrm{pg} / \mathrm{ml}$ for plasma metanephrine and $7.7 \%$ for $154 \mathrm{pg} / \mathrm{ml}$ and $8.9 \%$ for $2312 \mathrm{pg} / \mathrm{ml}$ for plasma normetanephrine. Assay sensitivity was $11 \mathrm{ng} / \mathrm{ml}$ for urinary metanephrine and $26 \mathrm{ng} / \mathrm{ml}$ for urinary

Table 1 Clinical characteristics of patients and volunteers. Values are means \pm S.E.M.

\begin{tabular}{|c|c|c|c|c|c|}
\hline Diagnosis & No. of patients & Age (years) & $\operatorname{Sex}(m / f)$ & Hypertension & Tumor size $(\mathrm{cm})$ \\
\hline PHEO & 24 & $41.7 \pm 3.2$ & $13 / 11$ & $92 \%$ & $3.9 \pm 0.5$ \\
\hline CPA & 21 & $52.8 \pm 3.3$ & $2 / 19$ & $67 \%$ & $3.3 \pm 0.2$ \\
\hline APA & 17 & $53.1 \pm 3.6$ & $5 / 12$ & $100 \%$ & $1.7 \pm 0.2$ \\
\hline NFM & 30 & $51.6 \pm 2.3$ & $15 / 15$ & $57 \%$ & $4.6 \pm 0.5$ \\
\hline $\mathrm{EH}$ & 16 & $43.0 \pm 3.6$ & $9 / 7$ & $100 \%$ & na \\
\hline VOL & 42 & $33.0 \pm 1.7$ & $28 / 14$ & $0 \%$ & na \\
\hline Total & 150 & $43.3 \pm 3.5$ & $72 / 78$ & na & na \\
\hline
\end{tabular}

PHEO, pheochromocytoma; CPA, cortisol-secreting adrenal adenoma; APA, aldosterone-secreting adrenal adenoma; NFM, nonfunctioning adrenal mass; $\mathrm{EH}$, essential hypertension; VOL, healthy volunteers; na, not available. 
normetanephrine. Assay variability was determined by within-assay coefficients of variation of $7.7 \%$ for $53 \mu \mathrm{g} / \mathrm{ml}$ and $4.2 \%$ for $201 \mu \mathrm{g} / \mathrm{ml}$ for urinary metanephrine and $11.1 \%$ for $248 \mu \mathrm{g} / \mathrm{ml}$ and $7.3 \%$ for $954 \mu \mathrm{g} / \mathrm{ml}$ for urinary normetanephrine. Betweenassay coefficients of variation were $10.4 \%$ for $71 \mu \mathrm{g} / \mathrm{ml}$ and $6.7 \%$ for $289 \mu \mathrm{g} / \mathrm{ml}$ for urinary metanephrine and $13.9 \%$ for $247 \mu \mathrm{g} / \mathrm{ml}$ and $7.8 \%$ for $881 \mu \mathrm{g} / \mathrm{ml}$ for urinary normetanephrine.

\section{Statistical evaluation}

Results are expressed as median and range. GraphPad Prism 3.0 (GraphPad Software Inc., San Diego, CA, USA) was used for statistical and receiver operating characteristic (ROC) analysis. Thresholds were established by ROC analysis. The Kruskal-Wallis test, followed by Dunn's multiple comparison test, were performed where appropriate. Sensitivity was calculated by a positive test result of patients with a pheochromocytoma divided by the total number of these patients. Specificity was obtained by a negative test result of patients with a surgically removed adrenal mass divided by the total number of these patients. ROC analyses included only patients with a surgically removed adrenal mass. Patients with essential hypertension and normotensive healthy volunteers were not considered. Corresponding 95\% confidence intervals as well as positive and negative likelihood ratios for the sensitivity and specificity are provided. A very high positive likelihood ratio $(\mathrm{LR}+)$ is defined by a value $>10$, high $\mathrm{LR}+$ is suggested by values between 5 and 10 , weak LR + is defined by values between 2 and 5 and very weak $\mathrm{LR}+$ is defined by values between 1 and 2. A very high negative likelihood ratio $(\mathrm{LR}-$ ) is reflected by values $<0.1$, high $\mathrm{LR}-$ is defined by values between 0.1 and 0.2 , weak LR is demonstrated by values between 0.2 and 0.5 and very weak LR - is suggested by values between 0.5 and 1 . When considering respective pairs of parameters (e.g. plasma metanephrine and normetanephrine), a true-positive test result was defined as patients with pheochromocytoma and at least one parameter over the threshold. A true-negative test result was defined as patients without pheochromocytoma and both parameters below the threshold. A false-negative test result was defined as patients with pheochromocytoma and normal measurements of both parameters. A falsepositive test result was defined as patients without pheochromocytoma and at least one elevated parameter. Analysis of correlations among tumor size and plasma and urinary parameters was carried out by the Spearman test. The reproducibility of the plasma metanephrine measurements was analyzed using the coefficient of variation. The results are expressed as means \pm s.E.M.

\section{Results}

\section{Plasma catecholamines}

Samples from twenty patients with pheochromocytoma and 62 control subjects were available for analysis. Epinephrine was $10.0 \mathrm{pg} / \mathrm{ml}(10-1780 \mathrm{pg} / \mathrm{ml})$ (median and range) in patients with pheochromocytoma (Table 2, Fig. 1), which was not significantly different to the other subgroups. ROC analysis revealed a threshold of $>24.5 \mathrm{pg} / \mathrm{ml}(0.134 \mathrm{nmol} / \mathrm{l})$ (sensitivity $40.0 \%$, specificity $88.7 \%$ ) for the diagnosis of pheochromocytoma using this parameter (Table 3, Fig. 2). LR + and LR - were weak and very weak respectively (Table 3). Norepinephrine was $378.5 \mathrm{pg} / \mathrm{ml}$ (10$10000 \mathrm{pg} / \mathrm{ml}$ ) in patients with pheochromocytoma (Table 2). It was significantly higher compared with patients with APA and hypertensive patients, but not significantly higher compared with any other subgroup (Table 2). ROC analysis provided a threshold of $>219.5 \mathrm{pg} / \mathrm{ml}(1.299 \mathrm{nmol} / \mathrm{l})$ (sensitivity 60.0\%, specificity $72.6 \%$ ) for the diagnosis of pheochromocytoma (Table 3, Fig. 2). LR + and LR - revealed a weak and very weak significance respectively (Table 3). Both parameters showed high specificity, but due to the low sensitivity a large number of patients with pheochromocytoma remained falsely undiagnosed (Fig. 1). When considering the combination of epinephrine and norepinephrine for the diagnosis of pheochromocytoma, the sensitivity increased to $70.0 \%$ with decreased specificity of $69.4 \%$ (Table 3).

\section{Urinary catecholamines}

Samples from fifteen patients with pheochromocytoma and 53 control subjects were available for analysis. Epinephrine was $7.4 \mu \mathrm{g} / 24 \mathrm{~h}(1-469 \mu \mathrm{g} / 24 \mathrm{~h})$ in patients with pheochromocytoma (Table 2). It was significantly higher than in patients with cortisol-secreting or non-functioning adenomas (Table 2). ROC analysis suggested a threshold of $2.5 \mu \mathrm{g} / 24 \mathrm{~h}$ $(0.014 \mu \mathrm{mol} / 24 \mathrm{~h}) \quad$ (sensitivity $73.3 \%$, specificity $64.2 \%$ ) (Table 3, Fig. 2). LR + and LR - reflected a rather weak significance. Norepinephrine was $112.6 \mu \mathrm{g} / 24 \mathrm{~h}(29.6-1000 \mu \mathrm{g} / 24 \mathrm{~h})$ in patients with pheochromocytoma, which was significantly higher than in all other subgroups except for patients with aldosterone-producing adenomas (Table 2). ROC analysis revealed an optimal threshold of $68.1 \mu \mathrm{g} / 24 \mathrm{~h}$ $(0.403 \mu \mathrm{mol} / 24 \mathrm{~h}) \quad$ (sensitivity $73.3 \%$, specificity $84.9 \%$ ) (Table 3, Fig. 2). Using this threshold, four pheochromocytomas remained undiagnosed (Fig. 1). $\mathrm{LR}+$ and LR - reflected a rather weak significance. A high clinical sensitivity $>95 \%$ decreased the specificity to $35.9 \%$ (Table 4). Combined use of urinary catecholamines increased the sensitivity to $93.3 \%$, but decreased the specificity to $58.5 \%$ in relation to the single parameters (Table 3). 


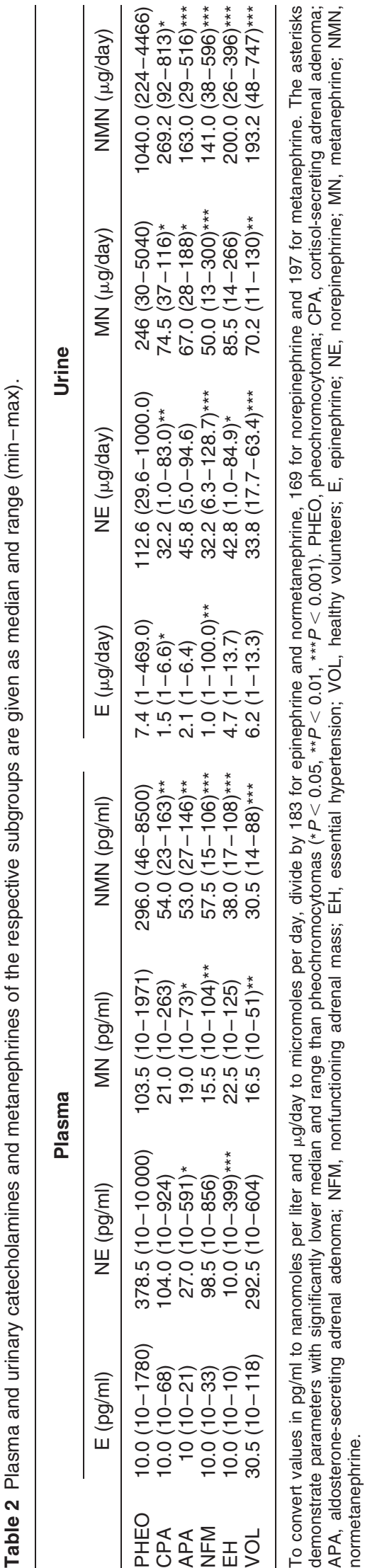

\section{Urinary metanephrines}

Samples from fifteen patients with pheochromocytoma and 52 control subjects were available for analysis. Metanephrine was $246 \mu \mathrm{g} / 24 \mathrm{~h}(30-5040 \mu \mathrm{g} / 24 \mathrm{~h})$ in patients with pheochromocytoma (Table 2). It was significantly higher compared with patients with APA, CPA, NFM and healthy volunteers (Table 2). ROC analysis revealed a threshold of $110.7 \mu \mathrm{g} / 24 \mathrm{~h}$ $(0.561 \mu \mathrm{mol} / 24 \mathrm{~h})$ for the diagnosis of pheochromocytoma (sensitivity $80.0 \%$, specificity $82.7 \%$ ) (Table 3, Fig. 2). LR + and LR - revealed a rather weak significance. A sensitivity $>95 \%$ revealed a specificity of 9.6\% (Table 4). Normetanephrine was $1040.0 \mu \mathrm{g} / 24 \mathrm{~h}$ $(224-4466 \mu \mathrm{g} / 24 \mathrm{~h})$ in patients with pheochromocytoma, which was significantly higher than in all other subgroups (Table 2). ROC analysis provided a threshold of $436.5 \mu \mathrm{g} / 24 \mathrm{~h}(2.383 \mu \mathrm{mol} / 24 \mathrm{~h})$ with high sensitivity $(93.3 \%)$ and specificity $(86.5 \%)$ for the diagnosis of pheochromocytoma (Table 3, Fig. 2). Only one pheochromocytoma remained below the threshold (Fig. 1). LR + and LR - demonstrated a high and very high significance respectively. A sensitivity $>95 \%$ decreased the specificity to $59.6 \%$ (Table 4). When considering the combination of urinary metanephrine and normetanephrine for the diagnosis of pheochromocytoma, a similar sensitivity of $93.3 \%$, but a lower specificity of $75.0 \%$ in relation to the single parameters was revealed (Table 3).

\section{Plasma metanephrines}

Samples from all twenty-four patients with pheochromocytoma and 68 control subjects were available for analysis. All patients satisfying the inclusion criterion underwent this index test. Metanephrine was $103.5 \mathrm{pg} / \mathrm{ml}(10-1971 \mathrm{pg} / \mathrm{ml})$ in patients with pheochromocytoma (Table 2). It was significantly higher compared with the remaining subgroups except for patients with CPA and hypertensive patients (Table 2). ROC analysis revealed a threshold of $38 \mathrm{pg} / \mathrm{ml}$ $(0.193 \mathrm{nmol} / \mathrm{l})$ (sensitivity $70.8 \%$, specificity $79.4 \%)$ for the diagnosis of pheochromocytoma (Table 3, Fig. 2). LR + and LR - revealed a weak significance. Seven patients with pheochromocytoma were falsely classified as negative (Fig. 1). Of these, six patients demonstrated elevated plasma normetanephrine levels. Falsely elevated measurements were detected in 20 patients. Three of these also had elevated normetanephrine levels. Normetanephrine was $296 \mathrm{pg} / \mathrm{ml}$ $(46-8500 \mathrm{pg} / \mathrm{ml})$ in patients with pheochromocytoma, which was significantly higher compared with all other groups (Table 2). ROC analysis suggested an optimal threshold of $125.5 \mathrm{pg} / \mathrm{ml}(0.686 \mathrm{nmol} / \mathrm{l})$ with very high sensitivity $(91.7 \%)$ and specificity $(95.6 \%)$ (Table 3, Fig. 2). The positive and negative likelihood ratios demonstrated very high diagnostic significance. Only two patients with pheochromocytoma remained 

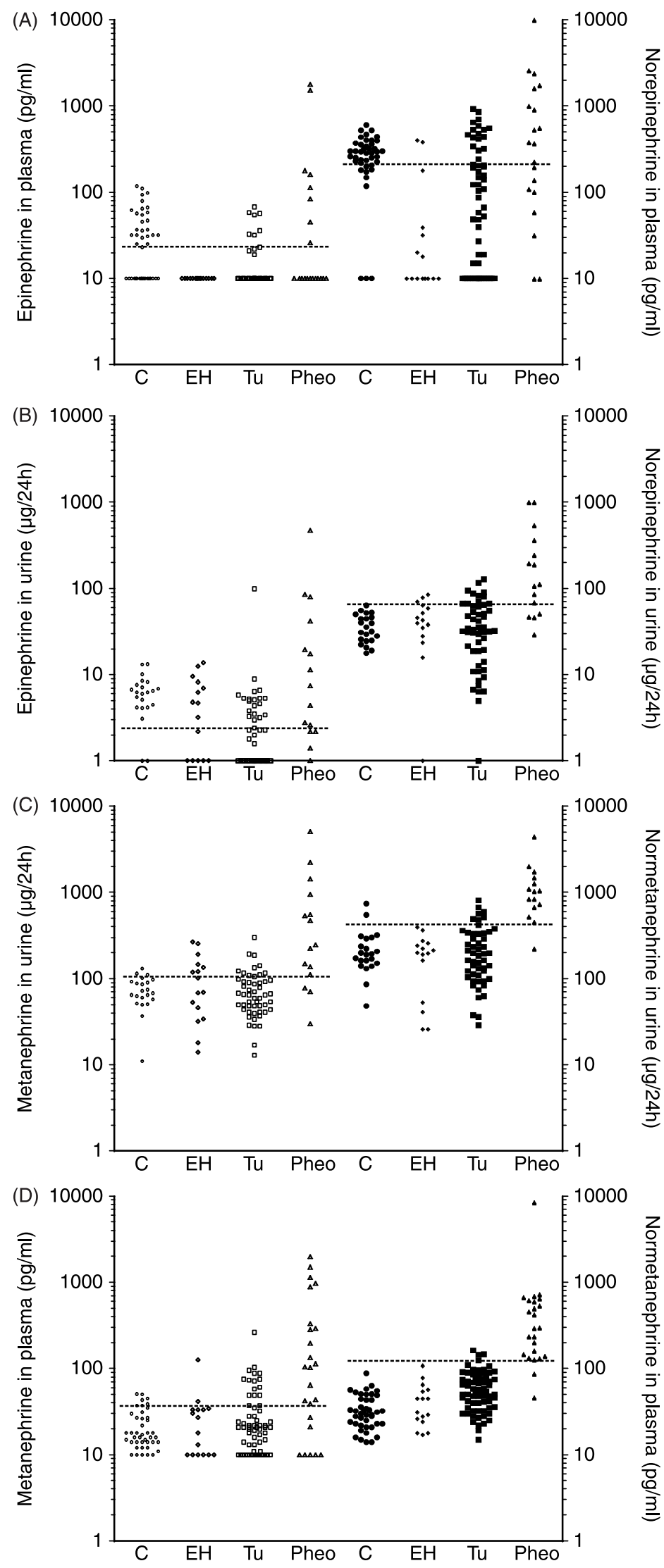

Figure 1 (A) Plasma and (B) urinary epinephrine and norepinephrine, $(C)$ urinary and (D) plasma metanephrine and normetanephrine in patients with pheochromocytoma (Pheo), adrenal mass (Tu), essential hypertension $(\mathrm{EH})$ and healthy volunteers $(\mathrm{C})$. The horizontal bar demonstrates the threshold for the diagnosis of pheochromocytoma calculated by ROC analysis. 
Table 3 Optimal thresholds for the single parameters and respective pairs determined by ROC analysis.

\begin{tabular}{|c|c|c|c|c|c|c|c|c|}
\hline & Threshold & Sens $(\%)(95 \% \mathrm{Cl})$ & False-neg. $(n)$ & Spec $(\%)(95 \% \mathrm{Cl})$ & False-pos. $(n)$ & LR+ & LR - & AUC $(95 \% \mathrm{Cl})$ \\
\hline \multicolumn{9}{|c|}{ Plasma (pg/ml) } \\
\hline$E$ & 24.5 & $40.0(19.12-63.95)$ & 12 & $88.7(78.11-95.34)$ & 7 & 3.54 & 0.70 & $0.64(0.48-0.79)$ \\
\hline NE & 219.5 & $60.0(36.05-80.88)$ & 8 & $72.6(59.77-83.15)$ & 17 & 2.19 & 0.59 & $0.73(0.60-0.86)$ \\
\hline$E+N E$ & $24.5 / 219.5$ & 70.0 & 6 & 69.4 & 19 & 1.65 & 0.51 & na \\
\hline MN & 38.0 & $70.8(48.91-87.38)$ & 7 & $79.4(67.88-88.26)$ & 14 & 3.44 & 0.29 & $0.76(0.62-0.89)$ \\
\hline NMN & 125.5 & $91.7(73.00-99.97)$ & 2 & $95.6(87.64-99.08)$ & 3 & 20.78 & 0.08 & $0.96(0.90-1.01)$ \\
\hline $\begin{array}{l}\text { MN + NMN } \\
\text { Urine }(\mu \mathrm{g} / \mathrm{d})\end{array}$ & $38.0 / 125.5$ & 95.8 & 1 & 79.4 & 14 & 4.82 & 0.05 & na \\
\hline $\mathrm{E}$ & 2.5 & $73.3(44.99-92.21)$ & 4 & $64.2(49.80-76.86)$ & 19 & 2.05 & 0.39 & $0.78(0.65-0.92)$ \\
\hline NE & 68.1 & $73.3(44.90-92.21)$ & 4 & $84.9(72.41-93.25)$ & 8 & 4.86 & 0.32 & $0.87(0.76-0.97)$ \\
\hline$E+N E$ & $2.5 / 68.1$ & 93.3 & 1 & 58.5 & 22 & 2.16 & 0.11 & na \\
\hline $\mathrm{MN}$ & 110.7 & $80.0(51.91-95.67)$ & 3 & 82.7 (69.67-91.77) & 9 & 4.62 & 0.30 & $0.86(0.74-0.99)$ \\
\hline NMN & 436.5 & $93.3(68.05-99.83)$ & 1 & $86.5(74.21-94.41)$ & 7 & 6.93 & 0.07 & $0.96(0.89-1.01)$ \\
\hline $\mathrm{MN}+\mathrm{NMN}$ & $110.7 / 436.0$ & 93.3 & 1 & 75.0 & 13 & 3.55 & 0.08 & na \\
\hline
\end{tabular}

To convert values in $\mathrm{pg} / \mathrm{ml}$ to nanomoles per liter and $\mu \mathrm{g} /$ day to micromoles per day, divide by 183 for epinephrine and normetanephrine, 169 for norepinephrine and 197 for metanephrine. The positive and negative likelihood ratios (LR) of plasma normetanephrine reflect very high diagnostic evidence. $\mathrm{E}$, epinephrine; NE, norepinephrine, MN, metanephrine; NMN, normetanephrine; Sens, sensitivity; neg., negative; Spec, specificity; pos., positive; LR+, positive likelihood ratio; LR - , negative likelihood ratio; Cl, confidence interval; AUC, area under the curve; na, not available.

below the threshold (Fig. 1). Of these, one patient presented with elevated plasma metanephrine levels. While none of the patients with essential hypertension or NFA and none of the healthy volunteers demonstrated elevated normetanephrine levels, three patients with CPA or APA had slightly increased levels. A sensitivity $>95 \%$ revealed a specificity of $77.9 \%$ (Table 4 .). Considering the combination of plasma metanephrine and normetanephrine for the diagnosis of pheochromocytoma demonstrated a higher sensitivity of $95.8 \%$, but a lower specificity of $79.4 \%$ (Table 3 ). Only one patient remained with normal plasma metanephrines. Four patients with CPA, two patients with APA, eight patients with NFA, one patient with hypertension and five healthy volunteers were falsely classified as positive. One of the CPA patients had a sevenfold higher plasma metanephrine level, while the remaining subjects demonstrated slightly elevated plasma metanephrines. The histological investigation postoperatively unequivocally confirmed a cortical adenoma in all patients with adrenal adenoma. No obvious drug interference was noticed. However, it cannot be ruled out in our investigation. Blood samples from nine healthy volunteers were collected on three consecutive days. The mean coefficient of variation was $12.3 \pm 1.6 \%$ for metanephrine and $14.0 \pm 2.0 \%$ for normetanephrine. Both demonstrated a high reproducibility for the plasma metanephrine measurements.

\section{Correlation among tumor size and plasma and urinary parameters}

A weak positive correlation was found between the tumor size of pheochromocytomas and plasma metanephrine $(r=0.56, P \leq 0.01)$ and urinary normetanephrine $(r=0.53, P \leq 0.05)$ (data not shown).

\section{Discussion}

This study compared catecholamines and metanephrines in plasma and urine for the diagnosis of pheochromocytoma in patients with adrenal tumors. For the first time, results are presented for plasma and urinary metanephrines measured by radioimmunoassay. ROC analysis revealed plasma normetanephrine as the best single parameter for the diagnosis of pheochromocytoma.

An increasing number of patients present with incidentally discovered adrenal masses. Therefore, reliable and simple parameters are required to exclude hormonally active tumors in these patients. Our study put special emphasis on patients with adrenal mass by including 68 adrenal tumors of various etiologies as controls. This group may not be truly representative of more typical patients tested for pheochromocytoma because of hypertensive signs and symptoms. Similarly, Raber et al. studied patients with pheochromocytoma and compared them with 14 patients with other adrenal tumors (7). In that study, normal values for plasma catecholamines and metanephrines were adapted from previous studies (8) and samples were measured by a chromatographic method. The authors demonstrated a sensitivity and specificity of $100 \%$ each for plasma normetanephrine.

In our study, twenty-four patients with histologically proven pheochromocytoma were included. Patients with surgically removed adrenal tumors of other etiologies served as control groups. Furthermore, hypertensive patients and normotensive, healthy volunteers were included, but were not considered for determination of thresholds. ROC analysis was employed to provide thresholds with an optimal balance between sensitivity and specificity. However, optimal thresholds by ROC analysis may demonstrate a low sensitivity, 

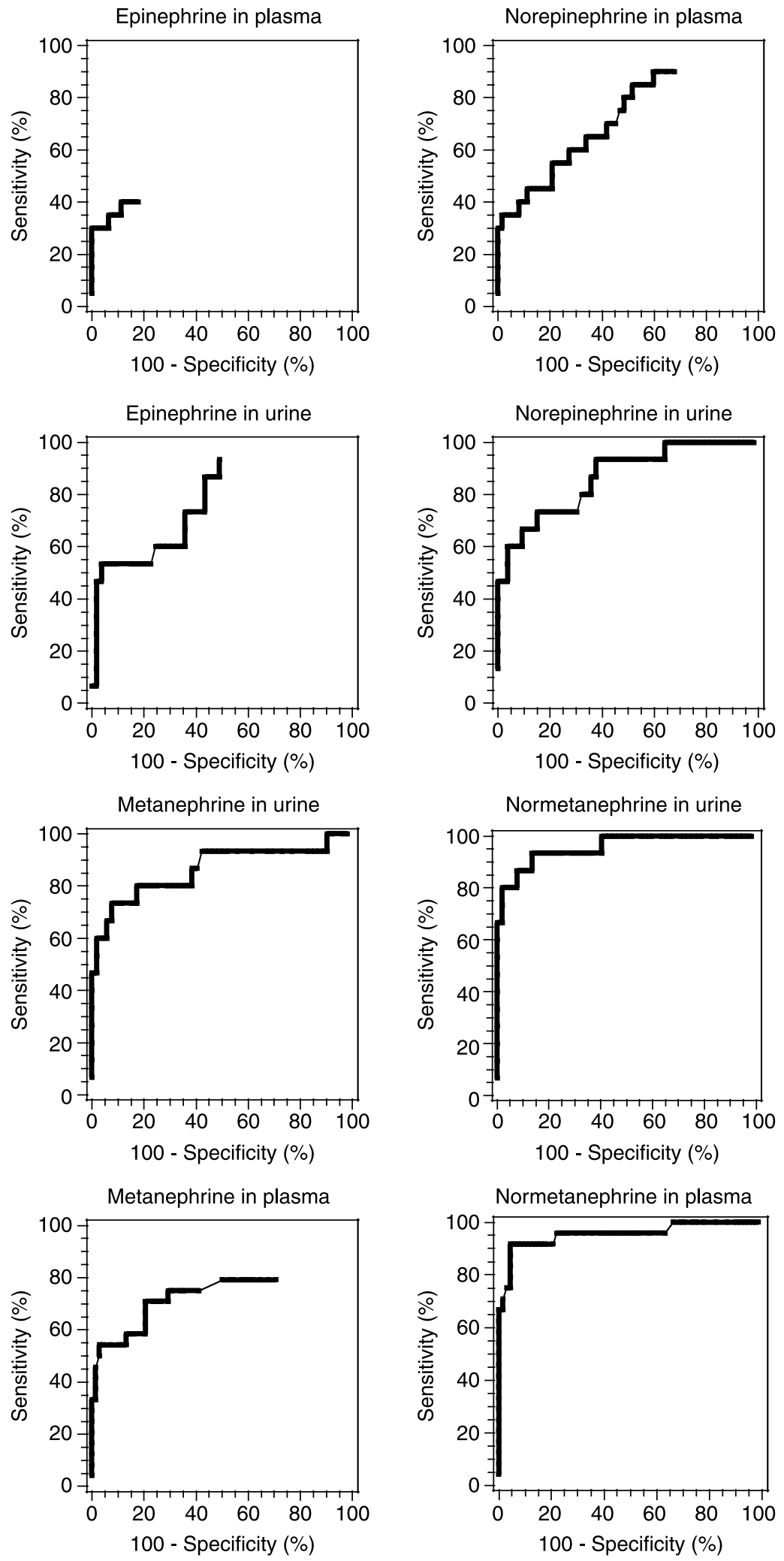

Figure $2 \mathrm{ROC}$ analysis curves for each of the eight parameters. 
Table 4 Thresholds with high clinical sensitivity (>95\%) and specificity ( $>95 \%)$ determined by ROC analysis.

\begin{tabular}{|c|c|c|c|c|c|c|c|c|}
\hline & $\begin{array}{c}\text { Threshold } \\
\text { (Sens }>95 \%)\end{array}$ & $\begin{array}{l}\text { Spec (\%) } \\
(95 \% \mathrm{Cl})\end{array}$ & LR+ & LR - & $\begin{array}{c}\text { Threshold } \\
(\text { Spec }>95 \%)\end{array}$ & $\begin{array}{l}\text { Sens (\%) } \\
(95 \% \mathrm{Cl})\end{array}$ & LR+ & LR - \\
\hline \multicolumn{9}{|c|}{ Plasma (pg/ml) } \\
\hline E & na & na & na & na & 56.0 & $30.0(11.89-54.28)$ & 6.2 & 0.74 \\
\hline NE & na & na & na & na & 674.5 & $35.0(15.39-59.22)$ & 7.23 & 0.68 \\
\hline MN & na & na & na & na & 92.0 & $54.2(32.82-74.45)$ & 12.28 & 0.48 \\
\hline NMN & 86.0 & $77.9(66.24-87.10)$ & 4.34 & 0.05 & 125.5 & $91.7(73.00-98.97)$ & 20.78 & 0.09 \\
\hline \multicolumn{9}{|c|}{ Urine $(\mu \mathrm{g} / \mathrm{d})$} \\
\hline $\mathrm{E}$ & na & na & na & na & 7.0 & $53.3(26.59-78.73)$ & 14.13 & 0.49 \\
\hline NE & 27.9 & $35.9(23.14-50.20)$ & 1.56 & 0 & 101.5 & $60.0(32.29-83.66)$ & 15.90 & 0.42 \\
\hline MN & 29.5 & $9.6(3.20-21.03)$ & 1.11 & 0 & 191.0 & $60.0(32.29-83.66)$ & 15.60 & 0.42 \\
\hline NMN & 221.5 & $59.6(45.10-72.99)$ & 2.48 & 0 & 633.5 & $80.0(51.91-95.67)$ & 20.80 & 0.21 \\
\hline
\end{tabular}

Limited by the sensitivity of the assay, a sensitivity $>95 \%$ was not available for plasma catecholamines and metanephrines and urinary epinephrine. E, epinephrine; NE, norepinephrine; MN, metanephrine; NMN, normetanephrine; Sens, sensitivity; Spec, specificity; LR + , positive likelihood ratio; LR - , negative likelihood ratio; $\mathrm{Cl}$, confidence interval; na, not available.

which is clinically not acceptable. A high sensitivity may be more important in the diagnosis of a potentially lethal condition such as pheochromocytoma. However, use of a high clinical sensitivity ( $\geq 95 \%)$ reflecting a reduced number of false-negative test results may be limited by very low specificity as demonstrated for some parameters in our study. The study was designed to analyze catecholamines and their metabolites in an outpatient setting. Therefore, samples were drawn by standard venipuncture, which may result in an increase in catecholamines and their metabolites.

This study revealed that plasma normetanephrine is the best single parameter. Using a threshold of $125.5 \mathrm{pg} / \mathrm{ml}$, only two patients were falsely negative. One of these presented preoperatively without clinical symptoms. Plasma catecholamines as well as plasma metanephrine were also negative. Urinary parameters were not available. Only the histological examination revealed a small $(1 \mathrm{~cm})$ pheochromocytoma in combination with an adenoma of the adrenal cortex. For the other patient, all biochemical markers were increased, except for normetanephrine in plasma.

When considering two respective parameters, plasma metanephrine and normetanephrine were the best biochemical combination compared with the other tests. Previous studies provided similar findings, with sensitivities between $97 \%$ and $100 \%$ and specificities between $85 \%$ and $96 \%$ using various thresholds $(8$, 9). However, most of these studies did not compare all four single parameters. An explanation for the potential superiority of metanephrines compared with catecholamines was provided by a recent study (10). Pheochromocytomas contain high amounts of catechol-O-methyltransferase (COMT), an enzyme that metabolizes catecholamines into free metanephrines. Rapid metabolism of catecholamines into metanephrines within the tumor may result in a continued high secretion of the metabolites, but an episodic and low secretion of the parent amines.

This study, for the first time, measured plasma metanephrines by radioimmunoassay, while previous studies employed chromatographic procedures (8). This might require different thresholds. A comparison of HPLC measurement of the same samples for plasma metanephrines is clearly of interest. This could not be applied in this study due to the small volume of the remaining samples. Whereas our threshold of $125.5 \mathrm{pg} / \mathrm{ml}$ for normetanephrine is quite similar to those of the previous studies using HPLC (range: $112-165 \mathrm{pg} / \mathrm{ml}$ ), a threshold of $38 \mathrm{pg} / \mathrm{ml}$ for metanephrine is rather low compared with thresholds between $59 \mathrm{pg} / \mathrm{ml}$ and $98 \mathrm{pg} / \mathrm{ml}$ in previous studies.

Patients included in this study were on different betablockers as well as on different central and peripheral alpha-blockers. Eisenhofer et al. demonstrated the possible influence of these drugs on the levels of catecholamines and their metabolites (11). Non-selective alpha-blockers may be associated with higher plasma and urinary levels of norepinephrine and normetanephrine, but not epinephrine and metanephrine. Beta-blockers, to a much lesser extent, may be responsible for elevated levels of plasma metanephrines and urinary catecholamines and metanephrines.

In our study, urinary fractionated metanephrines demonstrated a slightly lower sensitivity and specificity than their plasma counterparts. Urinary metanephrines largely reflect sulfate-conjugated metabolites, which are formed in gastrointestinal tissue. Thus, they are not related only to the pheochromocytoma, which may explain the reduced accuracy compared with plasma metanephrines $(12,13)$. Previous studies relied mostly on the measurement of urinary total metanephrines. In contrast to a separate determination of metanephrine and normetanephrine in urine, total metanephrines describe the combined measurement of metanephrine and normetanephrine in a single assay. Urinary total metanephrines demonstrated sensitivities between $65 \%$ and $89 \%$ and specificities between 89 and $95 \%(8,11,14,15)$. Determination of fractionated metanephrines measured by HPLC in previous studies resulted in higher sensitivities, from 88 to $97 \%$, but impaired 
specificities, from 69 to $85 \%(14,15)$. The thresholds established may not be applicable to pheochromocytomas due to genetically determined syndromes. Biochemical parameters were demonstrated to be higher in sporadic than in hereditary pheochromocytomas (14). Furthermore, in patients with pheochromocytoma and von-Hippel-Lindau syndrome only plasma normetanephrine concentrations were above normal (16). Our study included only patients with sporadic pheochromocytomas.

In conclusion, determination of plasma free metanephrine and normetanephrine, as measured by RIA, is a reliable test for the diagnosis of pheochromocytoma, especially in patients with adrenal tumors of unknown etiology.

\section{Acknowledgements}

We wish to thank DLD Diagnostika, Hamburg, Germany, for providing the assays for plasma and urinary metanephrine and normetanephrine. We also thank Helmut Tourne and Heike Sporkmann for skilful technical support.

\section{References}

1 Lenders JW, Eisenhofer G, Mannelli M \& Pacak K. Phaeochromocytoma. Lancet 2005366 665-675.

2 Eisenhofer G, Walther M, Keiser HR, Lenders JW, Friberg P \& Pacak K. Plasma metanephrines: a novel and cost-effective test for pheochromocytoma. Brazilian Journal of Medical and Biological Research 200033 1157-1169.

3 Eisenhofer G. Editorial: biochemical diagnosis of pheochromocytoma - is it time to switch to plasma-free metanephrines? Journal of Clinical Endocrinology and Metabolism $2003 \mathbf{8 8} 550-552$.

4 Roden M. How to detect pheochromocytomas? - the diagnostic relevance of plasma free metanephrines. Wiener Klinische Wochenzeitschrift $2002114246-251$.

5 Sawka AM, Prebtani AP, Thabane L, Gafni A, Levine M \& Young WF Jr. A systematic review of the literature examining the diagnostic efficacy of measurement of fractionated plasma free metanephrines in the biochemical diagnosis of pheochromocytoma. BMC Endocrine Disorders 200442.
6 Mundschenk J \& Lehnert H. Malignant pheochromocytoma. Experimental and Clinical Endocrinology and Diabetes 1998106 $373-376$.

7 Raber W, Raffesberg W, Bischof M, Scheuba C, Niederle B, Gasic S, Waldhausl W \& Roden M. Diagnostic efficacy of unconjugated plasma metanephrines for the detection of pheochromocytoma. Archives of Internal Medicine 2000160 2957-2963.

8 Lenders JW, Keiser HR, Goldstein DS, Willemsen JJ, Friberg P, Jacobs MC, Kloppenborg PW, Thien T \& Eisenhofer G. Plasma metanephrines in the diagnosis of pheochromocytoma. Annals of Internal Medicine 1995123 101-109.

9 Sawka AM, Jaeschke R, Singh RJ \& Young WF Jr. A comparison of biochemical tests for pheochromocytoma: measurement of fractionated plasma metanephrines compared with the combination of 24-hour urinary metanephrines and catecholamines. Journal of Clinical Endocrinology and Metabolism 200388 553-558.

10 Eisenhofer G, Keiser H, Friberg P, Mezey E, Huynh TT, Hiremagalur B, Ellingson T, Duddempudi S, Eijsbouts A \& Lenders JW. Plasma metanephrines are markers of pheochromocytoma produced by catechol-O-methyltransferase within tumors. Journal of Clinical Endocrinology and Metabolism 1998 $832175-2185$

11 Eisenhofer G, Goldstein DS, Walther MM, Friberg P, Lenders JW, Keiser HR \& Pacak K. Biochemical diagnosis of pheochromocytoma: how to distinguish true- from false-positive test results. Journal of Clinical Endocrinology and Metabolism $2003 \mathbf{8 8}$ $2656-2666$.

12 Eisenhofer G. Free or total metanephrines for diagnosis of pheochromocytoma: what is the difference? Clinical Chemistry 2001 47 988-989.

13 Eisenhofer G, Huynh TT, Hiroi M \& Pacak K. Understanding catecholamine metabolism as a guide to the biochemical diagnosis of pheochromocytoma. Reviews in Endocrine and Metabolic Disorders 20012 297-311.

14 Lenders JW, Pacak K, Walther MM, Linehan WM, Mannelli M, Friberg P, Keiser HR, Goldstein DS \& Eisenhofer G. Biochemical diagnosis of pheochromocytoma: which test is best? Journal of the American Medical Association 2002287 1427-1434.

15 Gardet V, Gatta B, Simonnet G, Tabarin A, Chene G, Ducassou D \& Corcuff JB. Lessons from an unpleasant surprise: a biochemical strategy for the diagnosis of pheochromocytoma. Journal of Hypertension $2001191029-1035$.

16 Eisenhofer G, Lenders JW, Linehan WM, Walther MM, Goldstein DS \& Keiser HR. Plasma normetanephrine and metanephrine for detecting pheochromocytoma in von Hippel-Lindau disease and multiple endocrine neoplasia type 2. New England Journal of Medicine 1999340 1872-1879.

Received 8 September 2005

Accepted 23 November 2005 